\title{
Preparation of EVAL membranes with smooth and particulate morphologies for neuronal culture
}

\author{
Tai-Horng Young ${ }^{\mathrm{a}, *}$, Chii-Wann Lin ${ }^{\mathrm{a}}$, Liao-Ping Cheng ${ }^{\mathrm{b}}$, Chih-Chen Hsieh ${ }^{\mathrm{a}}$ \\ ${ }^{\mathrm{a}}$ Institute of Biomedical Engineering, College of Medicine and College of Engineering, National Taiwan University, Taipei 10016, Taiwan \\ ${ }^{\mathrm{b}}$ Department of Chemical Engineering, Tamkang University, Taipei, Taiwan, ROC
}

Received 20 April 2000

\begin{abstract}
In this work, the in vitro interaction of cerebellar granule neurons prepared from 7-day-old Wistar rats and poly ethylene-co-vinyl alcohol (EVAL) membranes was investigated. Cells were cultured in smooth and particulate EVAL membranes for up to 7 days. Particulate membranes were prepared by using 1-octanol to precipitate EVAL solutions in DMSO. Such a membrane was microporous characterized by a packed bed of particles. Voids left between the aggregated particles formed a continuous and interconnected porous network. Crystallization of the EVAL polymer induced by 1-octanol is responsible for the formation of particulate morphology. The membrane structure and its relationship with cells were examined by scanning electron microscopy and the MTT assay. It was observed that the particulate membrane was more favorable for the neuron culture than the smooth membrane. Neurons seeded on the particulate membrane were able to regenerate with formation of an extensive neuritic network. Therefore, the particulate structure may spatially mediate cellular response that can promote neuronal cell attachment, differentiation and neuritic growth, indicating that the particulate structure should be useful as a new polymer scaffold for nerve repair. (C) 2001 Elsevier Science Ltd. All rights reserved.
\end{abstract}

Keywords: Poly(ethylene-co-vinyl alcohol) (EVAL); Particulate membranes; Neuron

\section{Introduction}

During the past several decades, various novel polymer membranes have experienced a notable growth in different phases of biomedical industry. Many techniques have been used to combine the membrane technology with different biological cells to fulfill specific purposes, such as islets for artificial pancreas [1-4], osteoblasts for membrane-guided bone regeneration $[5,6]$ and neuronal cells for nerve repair $[7,8]$. Especially, the neuronal cell behavior on a biomaterial has attracted much attention because of its scientific interest and clinical importance. Attempts to replace lost or dysfunctional neurons following trauma or disease by means of tissue transplantation or peripheral nerve grafting have been extensively investigated recently [9-12]. Obviously, the behavior of neuronal cells is influenced by both their intrinsic genetic

*Corresponding author. Tel.: + 8862-23123456 ext. 1455; fax: $+8862-23940049$.

E-mail address: thyoung@ha.mc.ntu.edu.tw (T.-H. Young). program and their extracellular environment. Therefore, the chemical nature and physical microstructure of the culture substrate can influence the behavior of neurons. Several studies have shown that modifications of the chemical structure and composition of the polymer substrates can greatly affect the degree of cell attachment and differentiation, and guidance of neuritic processes [13-19]. This research was developed to prepare synthetic polymer membranes with a special particulate morphology to improve the growth of neuronal cells. The particulate morphology was prepared by using 1-octanol to precipitate polyethylene-co-vinyl alcohol (EVAL) solutions in DMSO to form a membrane. Such a membrane was characterized by a packed bed of nearly equal-diameter particles approximately in the submicron order. It was proposed that crystallization of the polymer induced by 1-octanol is responsible for the formation of particulate morphology. Morphological studies of neurons seeded onto the particulate membrane demonstrated the capacity of such a membrane structure to support attachment and growth of neurons and neuritic growth. Therefore, this study developed an easy method to prepare 
membranes with the particulate structure for neuronal culture.

\section{Materials and methods}

\subsection{Membrane preparation}

Membranes were prepared by using EVAL (105A, Kuraray Co. Ltd., Japan) having an average ethylene content of $44 \mathrm{~mol} \%$. DMSO and 1-octanol of extra pure reagent grade (Nacalai Tesque, Kyoto, Japan) were used as received.

Two different structures of EVAL membrane were fabricated by using the dry and wet processes of the phase inversion method, respectively [20]. An appropriate amount of EVAL was dissolved in DMSO to form a $25 \mathrm{wt} \%$ homogeneous solution. This solution was dispersed uniformly on a glass plate $(\mathrm{ca} .100 \mu \mathrm{m})$ by an autocoater (KCC303, RK Print-Coat Instruments, UK). Subsequently, the membrane solution together with the glass plate were evaporated at $70^{\circ} \mathrm{C}$ to form dense membranes with a smooth morphology or directly immersed into 1-octanol to form porous membranes with a particulate morphology. DMSO and 1-octanol used in the preparation of EVAL membranes were potentially toxic for neurons and had to be extracted from the membranes. The smooth membrane was extensively washed by water and the particulate membrane was soaked in ethanol to remove 1-octanol and then in water to remove ethanol. Freeze-dried samples of the membranes were examined using a scanning electron microscope (SEM) to obtain the membrane structures.

\subsection{Gelation and liquid-liquid demixing boundaries}

To clearly understand the formation mechanism of EVAL membranes, knowledge of the equilibrium behavior in 1-octanol-DMSO-EVAL, which is related to different types of phase separation during the membrane formation, is required. The most straightforward method is to determine the crystallization-induced gelation and liquid-liquid demixing boundaries analyzed by the cloud point method as described in a previous publication [21,22]. Briefly, a specific amount of EVAL polymer (dried in oven at $50^{\circ} \mathrm{C}$ ) was mixed with a suitable amount of DMSO and 1-octanol mixture at $70^{\circ} \mathrm{C}$ until a clear homogeneous solution was obtained. The solution was then placed in an isothermal thermostat, which was maintained at $25^{\circ} \mathrm{C}$ for a period of 2 weeks. Two types of phase-separated results could be observed: (i) solution precipitated into a translucent or a white gel and (ii) solution became a clear liquid phase coexisting with a white solid. For case (i), the equilibrium gelation point was identified as the composition at which a sharp increase in turbidity was observed by a turbidity bridge
(Digital turbidimeter, Orbeco-Hellige, USA). The cloudy samples at these compositions are as a consequence of crystallization of EVAL molecules that have been proved by DSC scan in a previous publication [21,22]. For case (ii), gelation occurred in the polymer-rich phase after the liquid-liquid demixing [21,22]. Therefore, the liquid-liquid demixing and the gelation region would overlap in the phase diagram. The location of binodal in the ternary phase diagram was determined when the polymer-poor phase first occurred in a series of samples with increasing 1-octanol concentration.

\subsection{Cell culture}

EVAL membranes were cut into circular discs of $16 \mathrm{~mm}$ diameter suitably sized for tissue culture plate wells. The membranes were sterilized with $70 \%$ alcohol under ultraviolet light overnight and then rinsed extensively with phosphate buffer solution (PBS). Subsequently, membranes were placed in 24 well tissue culture polystyrene plates (Corning, New York, USA) by placing a silicon rubber ring on top of each membrane [23]. Before cell culture, membranes were covered with $10 \mathrm{mg} / 1$ poly-L-lysine (Sigma) [13] in PBS and incubated 2-3 h, after which time excess poly-L-lysine solution was removed by suction and dried for another hour. A glass coverslip (Assistent, Germany) was placed in 24 well tissue culture polystyrene plates as control, which was treated by the same way as test membrane-containing wells.

Cerebellar granule neurons were prepared from 7day-old Wistar rats according to Levi et al. [24]. Briefly, neurons were dissociated from freshly dissected cerebelli by mechanical disruption in the presence of trypsin and DNase. Following preparation, cells were seeded onto EVAL membranes and coverslips at a density of $4 \times 10^{5}$ cells/well in basal Eagle's medium (BME; Gibco) supplemented with $10 \%$ fetal calf serum (FCS; Gibco), $25 \mathrm{~mm} \mathrm{KCl}$, penicillin $\mathrm{G}(100 \mathrm{IU} / \mathrm{ml})$ and streptomycin $(100 \mu \mathrm{g} / \mathrm{ml})$. Cultures were maintained at $37^{\circ} \mathrm{C}$ in a humidified atmosphere of $95 \%$ air $/ 5 \% \mathrm{CO}_{2}$. Cytosine arabinoside $(10 \mu \mathrm{M})$ was added to the culture medium 18-24 $\mathrm{h}$ after plating to prevent replication of non-neuronal cells. Immunocytochemical analysis of these primary cultures has shown that they contain $95 \%$ granule neurons $[24,25]$.

\subsection{Assessment of neuronal viability}

The 3-(4,5-dimethylthiazol-2-yl)-2,5-diphenyl tetrazolium bromide (MTT, Sigma) was prepared as a $0.5 \mathrm{mg} / \mathrm{ml}$ stock solution in PBS, sterilized by Millipore filtration, and kept in dark [26]. MTT $(0.35 \mathrm{ml})$ was added to each well and plates were incubated for $5 \mathrm{~h}$ at $37^{\circ} \mathrm{C}$. Mitochodrial dehydrogenases of viable cells cleave selectively to 
the tetrazolium ring, yielding blue/purple formazan crystals. Therefore, the level of the reduction of MTT into formazan can reflect the level of cell metabolism. After incubation, the medium was aspirated and the formazan reaction products were dissolved in $0.4 \mathrm{ml}$ of $10 \%$ sodium dodecyl sulphate (SDS, Sigma) in PBS and the plates were then shaken for $15 \mathrm{~h}$ [27]. The optical density of the formazan solution was read on an ELISA plate reader (ELx 800, BIO-TEK) at $570 \mathrm{~nm}$. Cell viability determined by the MTT assay was performed at days 1, 3,5 and 7 after plating. All data were shown as the mean \pm standard deviation and statistical significance was assessed by Student's $t$-test. The level of significance was chosen as $p<0.05$.

For morphological observation, the cells adhering to the membrane were washed with PBS and then fixed with $2.5 \%$ glutaraldehyde in PBS for $1 \mathrm{~h}$ at $4{ }^{\circ} \mathrm{C}$. After thorough washing with PBS, the cells were dehydrated by graded ethanol changes and then critical point dried. Finally, the membranes were gold sputtered in vacuum and examined by SEM.

\section{Results}

\subsection{Membrane morphology}

Membranes prepared by evaporating DMSO at $70^{\circ} \mathrm{C}$ showed a fairly dense and smooth surface structure, as shown in Fig. 1. There were no pores existing in the top surface and cross section. In contrast, membranes prepared by precipitation in 1-octanol showed that the morphology was composed of submicron particles, as indicated in Fig. 2. Voids left between the aggregated particles formed a continuous and interconnected porous network. Therefore, a particulate and microporous substrate for neuronal culture was successfully prepared. The formation mechanism of particulate morphology can be explained by the phase diagram of 1-octanol-DMSOEVAL (shown in the next section). Basically, the particle structure is representative of crystallization from a homogeneous mixture [21,22] as have also been observed for other semicrystalline polymers $[28,29]$.

\subsection{The relation between the phase diagram and the membrane formation mechanism}

In Fig. 3, the phase diagram of 1-octanol-DMSOEVAL at $25^{\circ} \mathrm{C}$ is shown. The circular and triangular symbols were, respectively, the composition at which gelation and two equilibrium liquid phases first occurred in a series of samples with increasing 1-octanol concentration. A solution above the gelation boundary is homogeneous and transparent. EVAL polymer is dissolved in such a mixture at higher DMSO compositions. When the solution is within the crystallization-induced gelation gap, the solution will undergo solid-liquid demixing to form a gel consisting of small crystalline aggregates dispersed in a liquid phase [21,22]. Below the two equilibrium liquid data points, the supersaturated liquid is metastable with respect to liquid-liquid demixing. In general, initiation of liquid-liquid demixing is more rapid than nucleation of polymer crystallization that requires a rearrangement of polymer [30], so
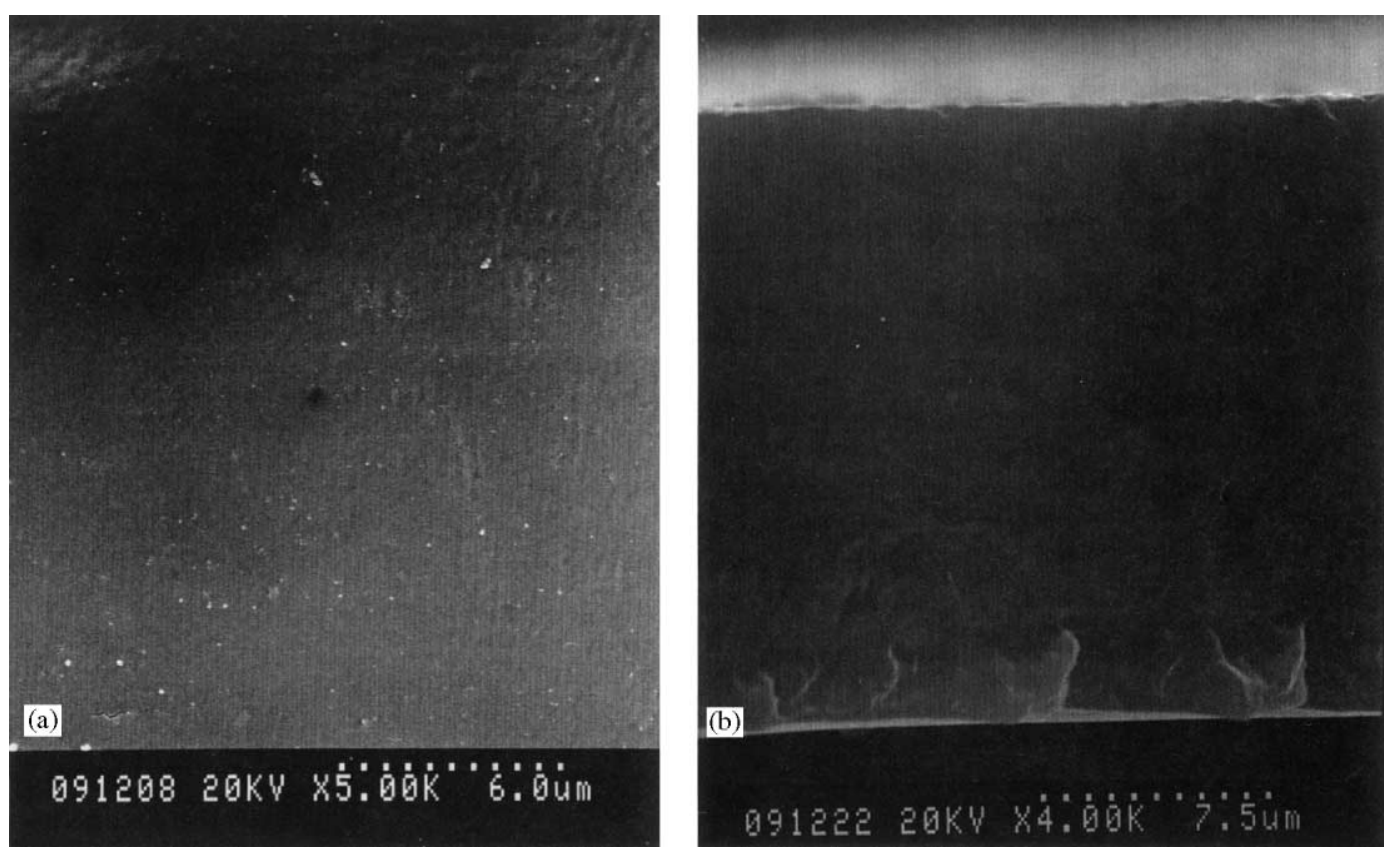

Fig. 1. Scanning electron microphotographs of the smooth EVAL membrane: (a) top surface view; (b) cross-sectional view. 

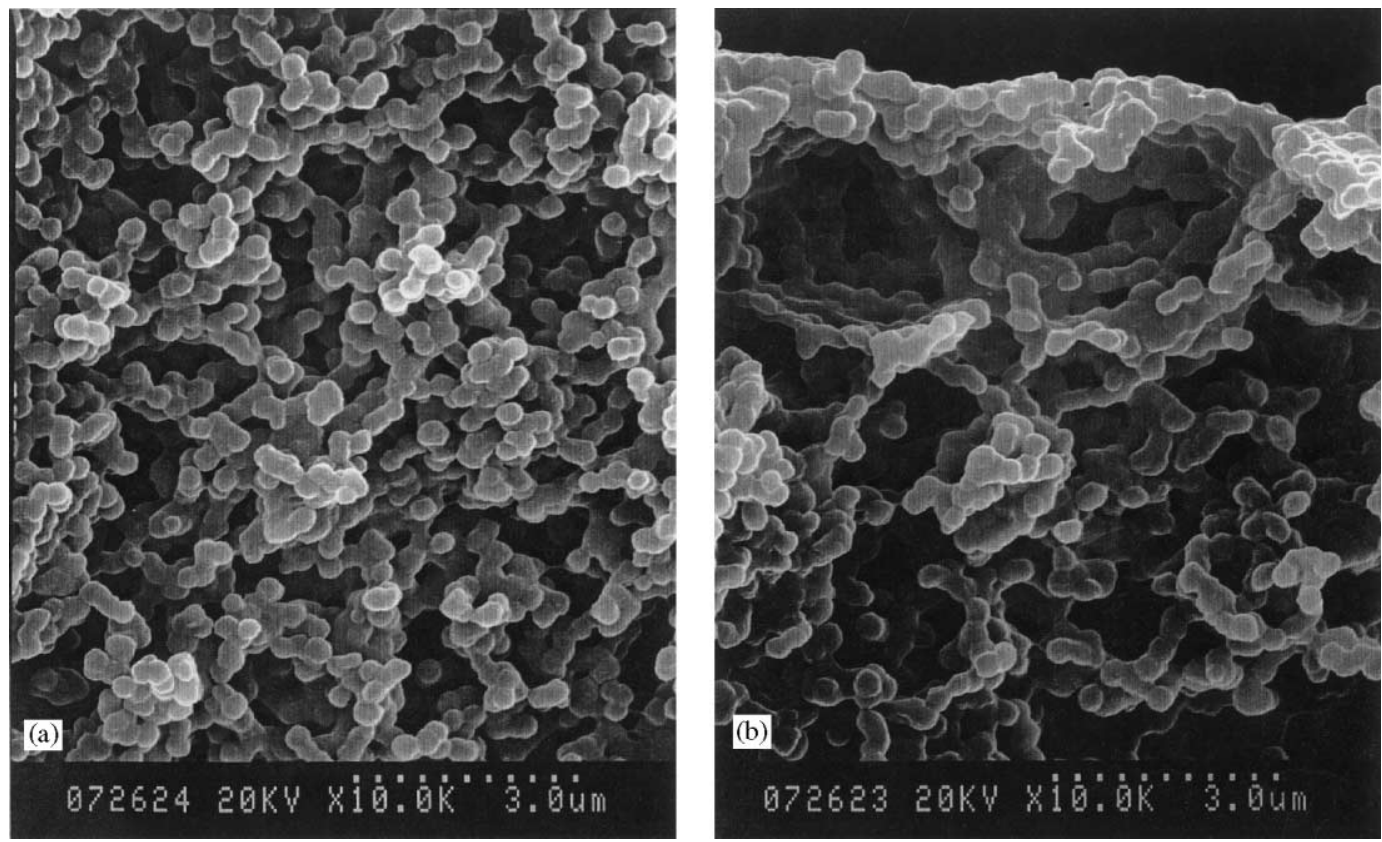

Fig. 2. Scanning electron microphotographs of the particulate EVAL membrane: (a) top surface view; (b) cross-sectional view.

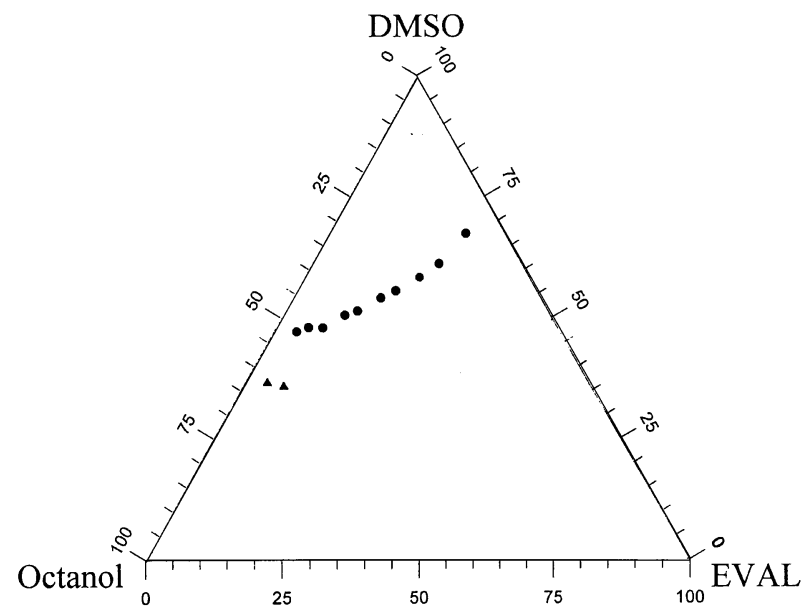

Fig. 3. Phase diagrams of 1-octanol-DMSO-EVAL at $25^{\circ} \mathrm{C}$. The data points, denoted by filled circles $(\mathbf{O})$ and triangles $(\mathbf{\Delta})$, represent the composition at which the gelation and two liquid phases first occurred in a series of samples with increasing 1-octanol concentration.

liquid-liquid demixing is kinetically favored to separate the supersaturated solution into two clear liquid phases. Subsequently, the polymer-rich phase will be always greatly supersaturated with respect to crystallization, thus, EVAL polymers eventually crystallize to form a white gel solid to coexist with a clear liquid phase. However, no liquid-liquid demixing data could be obtained experimentally at higher EVAL concentration in the phase diagram, as indicated in Fig. 3. This suggests that a highly supersaturated solution prefers to undergo the solid-liquid demixing rather than the liquid-liquid demixing. Therefore, using a $25 \mathrm{wt} \%$ EVAL solution to prepare membranes, the membrane structure with linking of particles is controlled by crystallization. Furthermore, the particles of this membrane are approximately of equal size, suggesting that all of the particles emerged from individual EVAL nuclei are born simultaneously. These nuclei then grow radially until their fronts meet and join with adjacent particles.

\subsection{Cell culture}

Fig. 4 shows the time course of formazan accumulation for the EVAL membranes and control. Formazan absorbance indicates that neurons seeded onto the EVAL membranes were able to convert the MTT into a blue formazan product. For the smooth membrane, the formation of formazan by neuronal cells exhibited a lag phase of 3 days, followed by an increase of up to day 7 . The formazan absorbance of control indicated a similar profile and only with a slight lower accumulation up to day 5 , but at a significantly lower level than that of the smooth EVAL membrane at day 7. This indicates that the smooth EVAL membrane can be used as a substrate for the attachment and growth of neuronal cells. In addition, a different trend in formazan deposition is observed between the particulate membrane and the smooth membrane. Examination of the kinetics of formazan production reveals that the particulate membrane had the highest accumulation of formazan and significant differences compared to control $(p<0.05)$ at all time points. The more formazan product present on the particulate membrane indicates particulate morphology was 


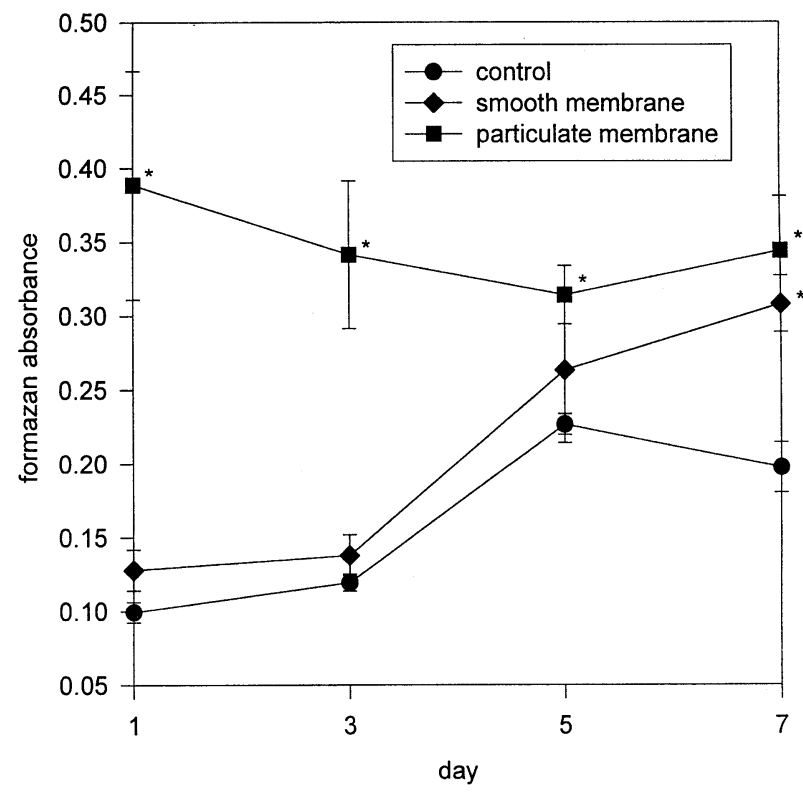

Fig. 4. MTT-tetrazolium assay. Formazan absorbance expressed as a function of time from cerebellar granule neurons seeded onto EVAL membranes and controls. Values are means \pm S.E.M. of 4 determinations.

favorable for the attachment of neuronal cells. The results of MTT assay were consistent with qualitative observations of SEM (shown in the next section). It is reasonable to hypothesize that the number of cell adhesion would increase as the area of its contact with the membrane surface increases. However, it is difficult to measure the surface area of a particulate membrane. In addition, it has been demonstrated that concave pores or convex particles on the membrane surface have a great influence on the behavior of myoblasts [31]. Therefore, exactly what factors are responsible for culturing neurons on the particulate membranes remains to be determined. Obviously, some direct influence of the surface particles on the neurons must be involved.

SEM of critical point dried membranes confirmed the presence of the neurons on the smooth and particulate EVAL membranes throughout the 7 days of culture (Figs. 5 and 6). In addition, astrocytes were also observed in Fig. 6(b), which was confirmed by using glial fibrillary acidic protein as a marker for astrocytes [32]. Although cytosine arabinoside was added to the culture medium to kill dividing non-neuronal cells that are synthesizing DNA $[24,25]$, some cells might survive because they were at stages of the cell cycle that were not going DNA synthesis. This indicates that EVAL membranes also have the potential as the substrate for culturing astrocytes.

Fig. 5 shows that most of the neurons displayed spherical or ellipsoid morphology on the smooth membrane. Some neurons formed aggregates on the smooth structure after 7 days in culture, but to a lesser extent than the

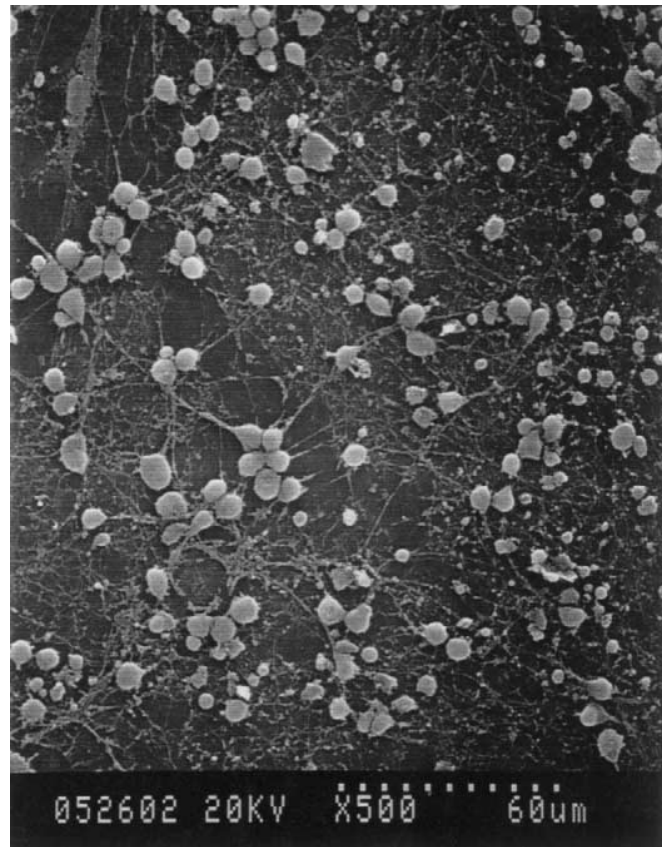

Fig. 5. Scanning electron microphotographs of neurons seeded onto the smooth EVAL membrane after 7 days in culture. The cells are round or ellipsoid in shape and formed aggregates with few neurites.

particulate structure cultures (Fig. 6). Furthermore, these cells already showed features of differentiation, with a few neurites that extended on the membrane surface. In contrast, although differences in cell morphology on membranes was not so clear, apparent large aggregates formed on the particulate membrane that varied in size from a few to several tens of cells. Qualitatively, the particulate membrane was more favorable for the neuron culture. Well-established neurons were prepared, so those cells interact with each other to form the cell pattern in aggregate cultures. In addition, on the particulate membrane, the density of the axonal network was more complex and dendritic arbors were more elaborate and highly branched. These aspects of development reflect the continuation of processes begun at a good stage of development. Therefore, the particulate membrane promoted cell-cell interaction for aggregate cultures, which led to the neuronal maturation and the development of a neural network.

\section{Discussion}

In this article, particulate membranes were easily prepared by using 1-octanol to precipitate EVAL solutions in DMSO. Based on the analysis of the phase diagram, the membrane structure with linking of particles is controlled by crystallization. Interestingly, besides EVAL, other crystallizable polymers such as Nylon-66 and 

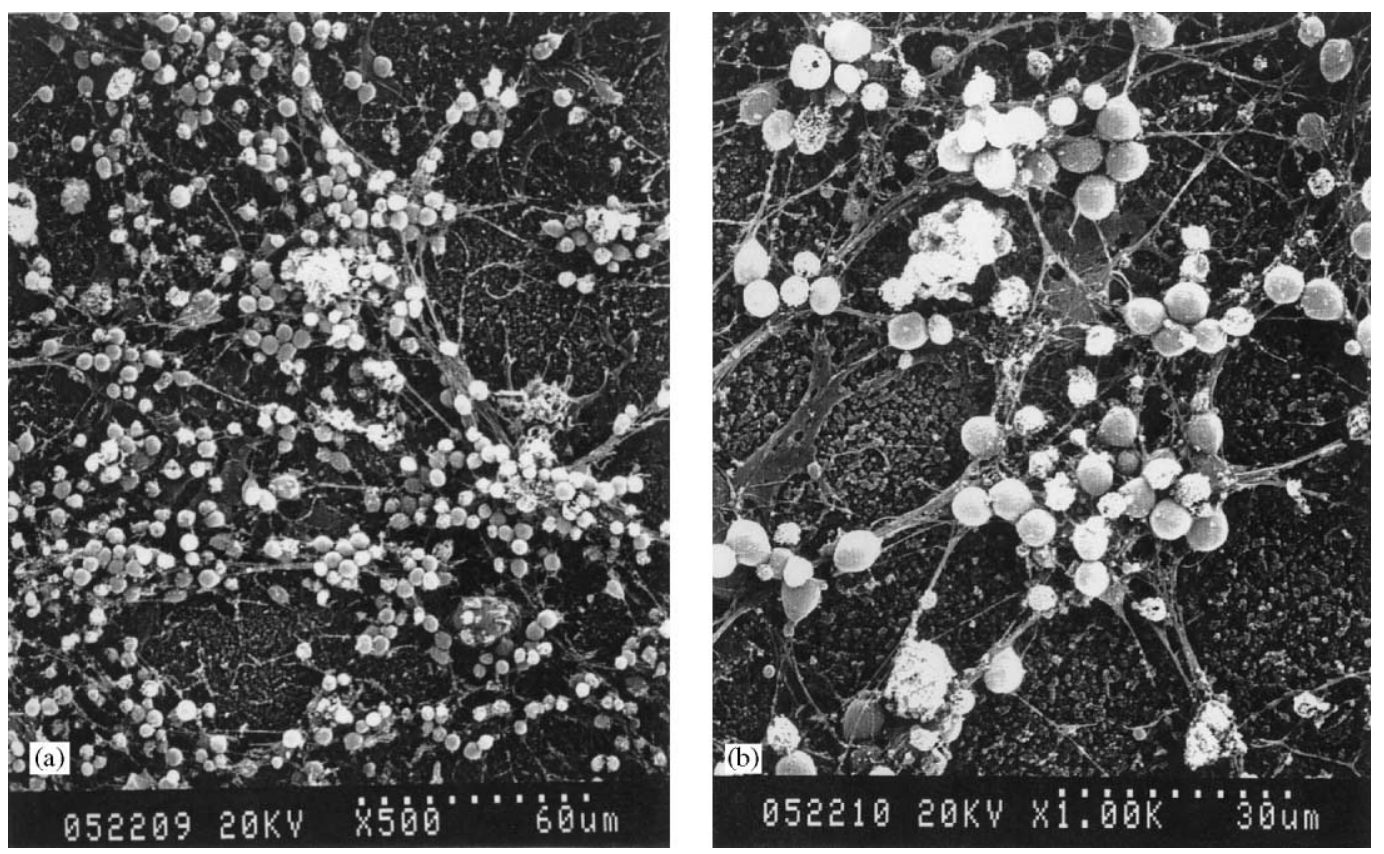

Fig. 6. Scanning electron microphotographs of neurons seeded onto the particulate EVAL membrane after 7 days in culture. (a) Most of cells have formed aggregates and showed neurites that form bundles. (b) A high magnification of some neurons attached directly to polymer particles.

PVDF can also form particulate membranes by precipitating polymer solutions in 1-octanol [33], In our laboratory, such particulate membranes have proved useful in plasma protein separation [33] and microfiltration [34]. However, prior to this study, very little was known about the characteristics of neuronal cell behavior on a particulate membrane. Therefore, we prepared EVAL membranes with smooth and particulate structures and studied the behavior of neurons on the different structure of EVAL membranes. The major advantage is that the behavior of neurons on different structures was effectively compared on the same material. The result clearly demonstrates that the membrane morphology is an important factor for attachment and growth of neurons. Although cell attachment would occur on both membrane surfaces, the neuritic network on the particulate surface was more complex compared to neuritic outgrowth on the smooth surface. In addition, the particulate morphology had a significant influence on cell aggregation. This suggests that cell aggregation can be influenced by the surface morphology of the substrate. Since the size of cell aggregate is controlled by cell migration [35], the particulate surface has a significant effect on cell motility. Therefore, although soluble neurotrophic factors and the chemical structure of the substrates contribute to the neuron growth, appropriate physical structures are also necessary for cell growth and integration.

Overall, the main finding of this study is the feasibility of culturing neuronal tissue cells on particulate EVAL membranes. These in vitro results are very encouraging since this information should be useful for the development of strategies for promoting the regrowth and regeneration of tissue in the nervous system. Such a particulate morphology can provide support structures upon which the neuronal cells grow and organize to form a tissue matrix permeable to axonal growth. Therefore, it has the potential to make use of particulate structure to improve healing and axonal regeneration of injured neural tissue and provide a means for the architectural design of more complex tissue-engineered systems. A thorough study of the effects of polymer surface structure properties on the attachment and differentiation of neurons will be followed by in vivo evaluation.

\section{Acknowledgements}

The authors thank the National Health Research Institute and Department of Health of the Republic of China for their financial support for the project DOH 87-HR-635.

\section{References}

[1] Lim F, Sun AM. Microencapsulated islets as bioartificial endocrine pancreas. Science 1980;210:908-10.

[2] Altman JJ, Houlbert A, Callard P, McMillan P, Solomon BA, Rosen J, Galetti PM. Long term plasma glucose normalization in experimental diabetic rats with macroencapsulated implants of benign human insulinomas. Diabetes 1986;35:625-33. 
[3] Young TH, Yao NK, Chang RF, Chen LW. Evaluation of asymmetric poly(vinyl alcohol) membranes for use in the artificial islets. Biomaterials 1996;17:2131-7.

[4] Young TH, Chuang WY, Yao NK, Chen LW. Use of a diffusion model for assessing the performance of poly(vinyl alcohol) bioartificial pancreases. J Biomed Mater Res 1998;40:385-91.

[5] Caffesse RG, Nasjleti CE, Morrison EC, Sanchez R. Guided tissue regeneration: comparison of bioabsorbable membranes. Histologic and histometric study in dogs. J Periodontol 1994;65:583-91.

[6] Nyman R, Magnusson M, Sennerby L, Nyman S, Lundgren D. Membrane-guided bone regeneration. Segmental radius defects studied in the rabbit. Acta Orthop Scand 1995;66:169-73.

[7] Woerly S, Marchand R, Lavallee C. Intracerebral implantation of synthetic polymer/biopolymer matrix: a new perspective for brain repair. Biomaterials 1990;11:97-107.

[8] Woerly S, Morassutti DJ. New aspects of neurotransplantation. Neurosurg Rev 1993;16:93-104.

[9] Aebischer P, Valentini RF, Dario P, Domenici C, Galletti PM. Piezoelectric guidance channels enhance regeneration in the mouse sciatic nerve after axotomy. Brain Res 1987;436:165-8.

[10] Valentini RF, Vargo TG, Gardella JA, Aebischer P. Electrically charged polymeric substrates enhance nerve fibre outgrowth in vitro. Biomaterials 1992;13:183-90.

[11] Langone F, Lora S, Veronese FM, Caliceti P, Parnigotto PP, Valenti F, Palma G. Peripheral nerve repair using a poly(organo)phosphazene tubular prosthesis. Biomaterials 1995;16:347-53.

[12] Shoichet MS, Winn SR, Athavale S, Harris JM, Gentile FT. Poly(ethylene oxide)-grafted thermoplastic membranes for use as cellular hybrid bio-artificial organs in the central nervous system. Biotech Bioeng 1994;43:563-72.

[13] Yavin E, Yavin Z. Attachment and culture of dissociated cells from rat embroy hemispheres on poly-lysine coated surfaces. J Cell Biol 1974;62:540-6.

[14] Carbonetto ST, Gruver MM, Turner DC. Nerve fiber growth on defined hydrogel substrates. Science 1982;216:897-9.

[15] Ruegg UT, Hefti F. Growth of dissociated neurons in culture dishes coated with synthetic polymeric amines. Neurosci Lett 1984;49:319-24.

[16] Ranieri JP, Bellamkonda R, Bekos EJ, Vargo TG, Gardella JA, Aebischer P. Neuronal cell attachment to fluorinated ethylene propylene films with covalently immobilized laminin oligopeptides YIGSR and IKVAN. II. J Biomed Mater Res 1995;29:779-85.

[17] Schugens Ch, Grandfils Ch, Jerome R, Teyssie Ph, Delree P, Martin D, Malgrange B, Moonen G. Preparation of a macroporous biodegradable polylactide implant for neuronal transplantation. J Biomed Mater Res 1995;29:1349-62.

[18] Woerly S, Marchand R, Lavallee C. Interactions of copolymeric poly(glyceryl methacrylate)-collagen hydrogels with neural tissue: effects of structure and polar groups. Biomaterials 1991;12:197-203.

[19] Woerly S, Maghami G, Duncan R, Subr V, Ulbrich K. Synthetic polymer derivatives as substrata for neuronal adhesion and growth. Brain Res Bull 1993;30:423-32.
[20] Kesting RE. Synthetic polymeric membranes. New York: Wiley, 1985.

[21] Young TH, Lai JY, Yu WM, Cheng LP. Equilibrium phase behavior of the membrane forming water-DMSO-EVAL copolymer system. J Membrane Sci 1997;128:55-65.

[22] Young TH, Cheng LP, Hsieh CC, Chen LW. Phase behavior of EVAL polymer in water-2-propanol cosolvent. Macromolecules 1998;31:1229-35.

[23] DeFife KM, Yun JK, Azeez A, Stack S, Ishihara K, Nakabayashi $\mathrm{N}$, Colton E, Anderson JM. Adhesion and cytokine production by monocyte on poly (2-methacryloyloxyethyl phosphorylcholineco-alkyl methacrylate)-coated polymers. J Biomed Mater Res 1995;29:431-9.

[24] Levi G, Aloisi F, Ciotti MT, Gallo V. Autoradiographic localization and depolarization-induced release of acidic amino acids in differentiating cerebellar granule cell cultures. Brain Res 1984; 290:77-86.

[25] Thangnipon W, Kingsbury A, Webb M, Balazs R. Observations on rat cerebellar cells in vitro: influence of substratum, potassium concentration and relationship between neurons and astrocytes. Dev Brain Res 1983;11:177-89.

[26] Mosmann T. Rapid colorimetric assay for cellular growth and survival: application of proliferation and cytotoxicity assays. J Immunol Methods 1983;65:55-63.

[27] Lau BHS, Li L, Yoon P. Thymic peptide protects vascular endothelial cells from hydrogen peroxide-induced oxidant injury. Life Sci 1993;52:1787-96.

[28] Lloyd DR, Kinzer KE, Tseng HS. Microporous membrane formation via thermally induced phase separation. I. Solid-liquid phase separation. J Membrane Sci 1990;52:239-61.

[29] Cheng LP, Young TH, Fang L, Gau JJ. Formation of particulate microporous PVDF membranes by isothermal immersion precipitation from 1-Octanol/Dimethylformamide/PVDF System. Polymer 1999;40:2395-403.

[30] Reuvers AJ, Altena FW, Smolders CA. Demixing and gelation behavior of ternary cellulose acetate solutions. J Polym Sci Polym Phys 1986;24:793-804

[31] Young TH, Yao CH, Sun JS, Lai CP, Chen LW. The effect of morphology variety of EVAL membranes on the behavior of myoblasts in vitro. Biomaterials 1998;19:717-24.

[32] Eng LF. Glial fibrillary acidic protein (GFAP): the major protein of glial intermediate filaments in differential astrocytes. J Neuroimmunol 1985;8:203-14.

[33] Lin DT, Cheng LP, Kang YJ, Chen LW, Young TH. Effects of precipitation conditions on the membrane morphology and permeation characteristics. J Membrane Sci 1998;140:185-94.

[34] Young TH, Cheng LP, Lin HY. Interesting behavior for filtration of macromolecules through EVAL membranes. Polymer 2000;41: $377-83$.

[35] Krewson CE, Chung SW, Dai W, Saltzman WM. Cell aggregation and neurite growth in gels of extracellular matrix molecules. Biotech Bioeng 1994;43:555-62. 